\title{
The reproductive solution for Fermat's Last theorem (elementary aspect)
}

\author{
PROF. DR. K. RAJA RAMA GANDHI ${ }^{1}$ AND REUVEN TINT ${ }^{2}$ \\ Resource person in Math for Oxford University Press and Professor at BITS-Vizag ${ }^{1}$ \\ Number Theorist, Israel ${ }^{2}$ \\ Email: editor126@gmail.com, reuven.tint@gmail.com
}

Abstract. We give a proof of the solvability in a natural numbers for Fermat's Last theorem and the equations

and

$$
a^{x}+b^{y}=c^{z}
$$

$$
\mathrm{X}_{1}^{\mathrm{n}_{1}}+\mathrm{X}_{2}^{\mathrm{n}_{2}}+\ldots+\mathrm{X}_{\mathrm{k}-1}^{\mathrm{n}_{\mathrm{k}-1}}=\mathrm{X}_{\mathrm{k}}^{\mathrm{n}_{\mathrm{k}}}
$$

has not been found earlier, significantly different from known, and allow us to obtain infinite set of solutions in natural numbers, and examples.

\section{Theorem 1.}

We must to prove that the equation

$$
\mathrm{x}^{\mathrm{n}}+\mathrm{y}^{\mathrm{n}}=\mathrm{z}^{\mathrm{n}}
$$

has no solutions in natural numbers (positive integers) for $n>2$.

Proof:

1.1. Suppose

$$
\mathrm{x}^{\prime}+\mathrm{y}^{\prime}=\mathrm{z}^{\prime}
$$

where $\mathrm{x}^{\prime}, \mathrm{y}^{\prime}-$ are arbitrary natural numbers, as

Then,

$$
x^{\alpha a-p b c>0}+y^{\beta b-q a c>0}=z^{\gamma c-m a b>0}
$$

$$
\alpha \mathrm{a}>\mathrm{pbc}, \beta \mathrm{b}>\text { qac, } \gamma \mathrm{c}>\mathrm{mab}
$$

1.1.1. Whenever

$$
\mathrm{p}=\alpha-1, \mathrm{q}=\beta-1, \mathrm{~m}=\gamma-1
$$

then

$$
\alpha<\frac{1}{1-\frac{a}{b c}}, \beta<\frac{1}{1-\frac{b}{a c}}, \gamma<\frac{1}{1-\frac{c}{a b}}
$$


For

$$
\begin{gathered}
\mathrm{a}=\mathrm{b}=\mathrm{c}=\mathrm{n} \\
\alpha=\beta=\gamma<\frac{1}{1-\frac{\mathrm{n}}{\mathrm{n}^{2}}}=\frac{\mathrm{n}}{\mathrm{n}-1}=\frac{2}{2-1}=2=1+\frac{1}{n-1}=1+\frac{1}{2-1} .
\end{gathered}
$$

Therefore, $\mathrm{n}=2$ and $\alpha=\beta=\gamma=1$, since the inequality is valid only for the case when comparing the elements of the same numerical system - the natural numbers. It follows that, ultimately, to the equation

(for $\alpha=\beta=\gamma=1$ and $a=b=c=n$ ):

$$
\mathrm{x}^{1 \times \mathrm{n}-(1-1) \times \mathrm{n}^{2}>0}+\mathrm{y}^{1 \times \mathrm{n}-(1-1) \times \mathrm{n}^{2}>0}=\mathrm{z}^{1 \times \mathrm{n}-(1-1) \times \mathrm{n}^{2}>0}
$$

and since " $n "$ is not greater than two, that lead to equations

$$
\mathrm{x}^{2}+\mathrm{y}^{2}=\mathrm{z}^{2} \text { and } \mathrm{x}+\mathrm{y}=\mathrm{z}
$$

1.1.2. If

$$
\alpha \mathrm{a}>(\alpha-\mathrm{k}) \mathrm{bc}
$$

then

$$
\alpha(\mathrm{bc}-\mathrm{a})<\mathrm{kbc}
$$

and

$$
\alpha<\mathrm{k} \times \frac{b c}{b c-a}=k \times \frac{n}{n-1}(k>1) .
$$

Multiply the two numbers " $\mathrm{k}$ " and $\frac{n}{n-1}$ in the system of natural numbers for $(n>2)$ is incorrectly.

1.1.3. If

$$
\mathrm{b}=\mathrm{c}=\mathrm{n},
$$

then

$$
\alpha \mathrm{a}-\mathrm{pn}^{2}>0
$$

and

$$
\mathrm{p}<\frac{\alpha a}{n^{2}}
$$

For $\mathrm{a}=\mathrm{n} \quad \mathrm{p}<\alpha \times \frac{1}{n}$

Multiply the two numbers $\alpha$ and $\frac{1}{n}$ in the system of natural numbers $\mathrm{n}>1$ is incorrectly.

Sets of functions $\frac{1}{n}$ and $\frac{n}{n-1}$ are not closed under superposition. 
1.2. It is easy to verify, that if

$$
\mathrm{x}+\mathrm{y}=\mathrm{z}
$$

then

$$
\alpha \mathrm{a}-\mathrm{pbc}=1, \beta \mathrm{b}-\mathrm{qac}=1, \gamma \mathrm{c}-\mathrm{mab}=1,
$$

where

$$
(\alpha \mathrm{a}, \mathrm{pbc})=1,(\beta \mathrm{b}, \mathrm{qac})=1,(\gamma \mathrm{c}, \mathrm{mab})=1,(\alpha, \mathrm{p})=1,(\beta, \mathrm{q})=1,(\gamma, \mathrm{m})=1
$$

- are relatively prime integers, $\mathrm{a}, \mathrm{b}, \mathrm{c}$ - are pairwise relatively prime integers(having no common factor other than one).

1.3.

$$
\alpha \mathrm{a}-\mathrm{pbc}=\beta \mathrm{b}-\mathrm{qac}=\gamma \mathrm{c}-\mathrm{mab}=0
$$

is impossible since

$$
1+1 \neq 1
$$

1.4. If

$$
\begin{gathered}
\alpha=\beta=\gamma=\mathrm{p}=\mathrm{q}=\mathrm{m}=1 \\
\mathrm{a}>\mathrm{bc}, \mathrm{b}>\mathrm{ac}, \mathrm{c}>\mathrm{ab} \text { and } 1>\mathrm{a}^{2}, 1>\mathrm{b}^{2}, 1>\mathrm{c}^{2},
\end{gathered}
$$

then it is impossible.

1.5. Thus, consider all possible variants for our case the solutions of equation

$$
\mathrm{x}+\mathrm{y}=\mathrm{z}
$$

1.6. Therefore, the equation

$$
\mathrm{x}^{\mathrm{n}}+\mathrm{y}^{\mathrm{n}}=\mathrm{z}^{\mathrm{n}}
$$

is not solvable in natural numbers for $\mathrm{n}>2$ as required. This completes the proof.

This is an elementary proof, with a high degree of probability could be obtained by Fermat.

2.1. Multiplying [1] by

$$
\mathrm{t}=\mathrm{x}^{\mathrm{pbc}} \times \mathrm{y}^{\mathrm{qac}} \times \mathrm{z}^{\mathrm{mab}} .
$$

Given

$$
\left(x^{\alpha} \times y^{q c} \times z^{m b}\right)^{a}+\left(x^{p c} \times y^{\beta} \times z^{m a}\right)^{b}=\left(x^{p b} \times y^{q a} \times z^{\gamma}\right)^{c} .
$$

2. 2.

$$
\mathrm{a}=4, \mathrm{~b}=5, \mathrm{c}=7 \text {, }
$$




$$
\begin{gathered}
\alpha \times 4-\mathrm{p} \times 5 \times 7=1, \mathrm{p}=1, \alpha=9 \\
\beta \times 5-\mathrm{q} \times 4 \times 7=1, \mathrm{q}=3 ; \beta=17 \\
\gamma \times 7-\mathrm{m} \times 4 \times 5=1, \mathrm{~m}=1, \gamma=3, \\
\left(\mathrm{x}^{9} \times \mathrm{y}^{21} \times \mathrm{z}^{5}\right)^{4}+\left(\mathrm{x}^{7} \times \mathrm{y}^{17} \times \mathrm{z}^{4}\right)^{5}=\left(\mathrm{x}^{5} \times \mathrm{y}^{12} \times \mathrm{z}^{3}\right)^{7} \\
\text { as } \mathrm{x}+\mathrm{y}=\mathrm{z} .
\end{gathered}
$$

2.3. If

$$
3^{2}+4^{2}=5^{2}
$$

then

$$
\left[3 \times\left(3^{35} \times 4^{84} \times 5^{20}\right)\right]^{2}+\left[4 \times\left(3^{35} \times 4^{84} \times 5^{20}\right)\right]^{2}=\left[5 \times\left(3^{35} \times 4^{84} \times 5^{20}\right)\right]^{2}
$$

and

$$
\left[\left(3^{9} \times 4^{21} \times 5^{5}\right)^{2}\right]^{4}+\left[\left(3^{7} \times 4^{17} \times 5^{4}\right)^{2}\right]^{5}=\left[\left(3^{5} \times 4^{12} \times 5^{3}\right)^{2}\right]^{7}
$$

- In fact, there is a slightly modified solution of L. Yushmanovich -(6, page 74).

2.4. Using the equation $[2]$, if

$$
\alpha_{0}, \beta_{0}, \gamma_{0}, \rho_{0}, q_{0}, m_{0}
$$

are any (or minimal solution) related above equations in natural numbers for fixed values $a, b, c$ ,then

$$
\begin{array}{cl}
\alpha=\alpha_{0}+\mathrm{bc} \theta_{1} & , \rho=\rho_{0}+\mathrm{a} \theta_{1}, \\
\beta=\beta_{0}+\mathrm{ac}_{2} & , \mathrm{q}=\mathrm{q}_{0}+\mathrm{b} \theta_{2}, \\
\gamma=\gamma_{0}+\mathrm{ab}_{3} & , \mathrm{~m}=\mathrm{m}_{0}+\mathrm{c} \theta_{3},
\end{array}
$$

$\theta_{1}, \theta_{2}, \theta_{3}$ - are arbitrary natural (whole) numbers or zero, and

$$
\begin{aligned}
& \left(\mathrm{x}^{\alpha_{0}+\mathrm{bc} \theta_{1}} \times \mathrm{y}^{\mathrm{q}_{0} c+b c \theta_{2}} \times \mathrm{z}^{\mathrm{m}_{0} b+b c \theta_{3}}\right)^{a}+ \\
& +\left(\mathrm{x}^{\mathrm{p}_{0} \mathrm{c}+\mathrm{ac} \theta_{1}} \times \mathrm{y}^{\beta_{0}+a c \theta_{2}} \times \mathrm{z}^{\mathrm{m}_{0} a+a c \theta_{3}}\right)^{b}= \\
& =\left(\mathrm{x}^{\mathrm{p}_{0} \mathrm{~b}+\mathrm{ab} \theta_{1}} \times \mathrm{y}^{\mathrm{q}_{0} a+a b \theta_{2}} \times \mathrm{z}^{\gamma_{0}+a b \theta_{3}}\right)^{c} .
\end{aligned}
$$

2.5.

$$
\begin{gathered}
a=4 ; b=5 ; c=7 \\
\alpha=9+5 \times 7=44 ; \rho=1+4=5
\end{gathered}
$$




$$
\begin{gathered}
\beta=17+4 \times 7=45 ; \mathrm{q}=3+5=8 \\
\gamma=3+4 \times 5=23 ; \mathrm{m}=1+7=8 \\
\left(\mathrm{x}^{44} \times \mathrm{y}^{56} \times \mathrm{z}^{40}\right)^{4}+\left(\mathrm{x}^{35} \times \mathrm{y}^{45} \times \mathrm{z}^{32}\right)^{5}= \\
=\left(\mathrm{x}^{25} \times \mathrm{y}^{32} \times \mathrm{z}^{23}\right)^{7}
\end{gathered}
$$

2.6. If

$$
\mathrm{x}_{1}+\mathrm{x}_{2}+\ldots .+\mathrm{x}_{k-1}=\mathrm{x}_{k}
$$

then

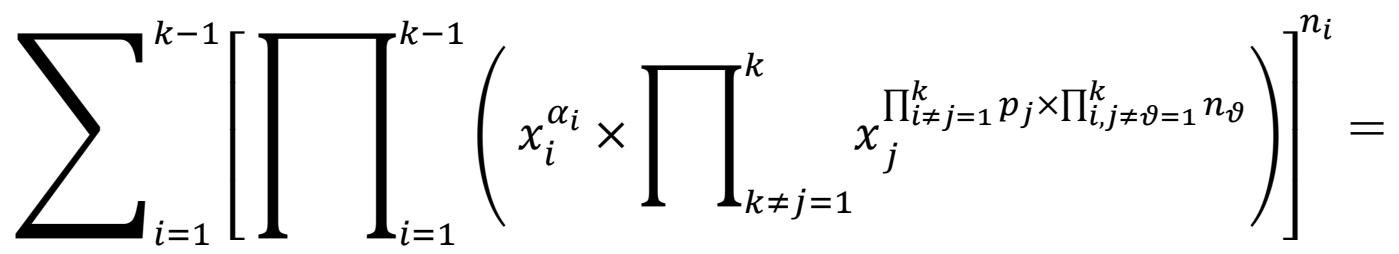

$$
\begin{aligned}
& =\left(x_{k}^{\alpha_{k}} \times \prod_{k \neq j=1}^{k-1} x_{j}^{\prod_{k \neq j=1}^{k-1} p_{j} \times \prod_{k, k-1 \neq \vartheta=1}^{k-2} n_{\vartheta}}\right)^{n_{k}}
\end{aligned}
$$

where

$\mathrm{x}_{1}, \mathrm{x}_{2}, \ldots ., \mathrm{x}_{\mathrm{k}-1}-$ are arbitrary natural numbers;

$\mathrm{x}_{\mathrm{k}}=\sum_{\mathrm{i}=1}^{\mathrm{k}-1} \mathrm{x}_{\mathrm{i}}$

$\mathrm{n}_{1}, \mathrm{n}_{2}, \ldots, \mathrm{n}_{\mathrm{k}-1}-$ are pairwise relatively prime arbitrary natural numbers,

$k \geq 3$

$i=1,2, \ldots ., \mathrm{k}-$ are numbers of brackets;

$\mathrm{j}=1,2, \ldots ., \mathrm{k}$ - are numbers inside brackets;

$\sum, \Pi$ - are signs of sum and products;

2.7. If given $\mathrm{n}_{i}$ and $\mathrm{n}_{k}$ of " $\mathrm{k}$ " values, then $\alpha_{i}$ and $\mathrm{p}_{j}$ determined by the " $\mathrm{k}$ " in the following equations:

$$
\alpha_{i} n_{i}-\rho_{j} \prod_{i \neq j=1}^{k} n_{j}=1
$$


2.8. For example, for $\mathrm{k}=4$

$$
\begin{aligned}
& \left(x_{1}^{\alpha_{1}} \times x_{2}^{p_{2} n_{3} n_{4}} \times x_{3}^{p_{3} n_{2} n_{3}} \times x_{4}^{p_{4} n_{2} n_{3}}\right)^{\mathrm{n}_{1}}+ \\
+ & \left(x_{2}^{\alpha_{2}} \times x_{1}^{p_{1} n_{3} n_{4}} \times x_{3}^{p_{3} n_{1} n_{4}} \times x_{4}^{p_{4} n_{1} n_{3}}\right)^{\mathrm{n}_{2}}+ \\
+ & \left(x_{3}^{\alpha_{3}} \times x_{1}^{p_{1} n_{2} n_{4}} \times x_{2}^{p_{2} n_{1} n_{4}} \times x_{4}^{p_{4} n_{1} n_{2}}\right)^{\mathrm{n}_{3}}= \\
= & \left(x_{4}^{\alpha_{4}} \times x_{1}^{p_{1} n_{2} n_{3}} \times x_{2}^{p_{2} n_{1} n_{3}} \times x_{3}^{p_{3} n_{1} n_{2}}\right)^{\mathrm{n}_{4}}
\end{aligned}
$$

, and if

$$
\begin{gathered}
\mathrm{n}_{1}=3, \mathrm{n}_{2}=4, \mathrm{n}_{3}=5, \mathrm{n}_{4}=7 \\
47 \times 3-1 \times 4 \times 5 \times 7=1 \\
79 \times 4-3 \times 3 \times 5 \times 7=1 \\
17 \times 5-1 \times 3 \times 4 \times 7=1 \\
43 \times 7-5 \times 3 \times 4 \times 5=1 . \\
\alpha_{1}=47, \alpha_{2}=79, \alpha_{3}=17, \alpha_{4}=43 \\
\mathrm{p}_{1}=1, \mathrm{p}_{2}=3, \mathrm{p}_{3}=1, \mathrm{p}_{4}=5 \\
\left(x_{1}^{47} \times x_{2}^{105} \times x_{3}^{28} \times x_{4}^{100}\right)^{3}+\left(x_{2}^{79} \times x_{1}^{35} \times x_{3}^{21} \times x_{4}^{75}\right)^{4}+ \\
+\left(x_{3}^{17} \times x_{1}^{28} \times x_{2}^{63} \times x_{4}^{60}\right)^{5}=\left(x_{4}^{43} \times x_{1}^{20} \times x_{2}^{45} \times x_{3}^{12}\right)^{7} .
\end{gathered}
$$

2.9. If

$$
27^{5}+84^{5}+110^{5}+133^{5}=144^{5}
$$

- is well-known solution, then

$$
\begin{aligned}
& \left(27^{1502} \times 84^{5005} \times 110^{5148} \times 133^{819} \times 144^{2541}\right)^{5 \times 2}+ \\
+ & \left(27^{1001} \times 84^{3337} \times 110^{3432} \times 133^{546} \times 144^{1694}\right)^{5 \times 3}+ \\
+ & \left(27^{429} \times 84^{1430} \times 110^{1471} \times 133^{234} \times 144^{726}\right)^{5 \times 7}+ \\
+ & \left(27^{273} \times 84^{910} \times 110^{936} \times 133^{149} \times 144^{462}\right)^{5 \times 11}= \\
& =\left(27^{231} \times 84^{770} \times 110^{792} \times 133^{126} \times 144^{391}\right)^{5 \times 13}
\end{aligned}
$$

- In fact, there is a slightly modified solution of L. Yushmanovich

for arbitrary natural numbers " $\mathrm{n} "$ and arbitrary dimensions $(\mathrm{k} \geq 3)-(6)$, стр.74. 


\section{References:}

[1] H.Davenport, "The Higher Arithmetic", Moscow, 1965.

[2] V. Sierpiński, "250 Problems in Elementary Number Theory”, Moscow, 1968.

[3] U. Davidov, " Problems and exercises in theoretical arithmetic of whole numbers ", Minsk, 1963.

[4] A. Kurosh, “Higher Algebra", "Science”, Fizmatgiz, Moscow, 1971.

[5] Н.Н. Воробьев, "Признаки делимости”, “Science”, Fizmatgiz, Moscow, 1974.

[6] V. Sierpiński, “О решении уравнений в целых числах”, Fizmatgiz, Moscow, 1961. 